\title{
Desmoplastic Infantile Astrocytoma
}

National Cancer Institute

\section{Source}

National Cancer Institute. Desmoplastic Infantile Astrocytoma. NCI Thesaurus. Code C9476.

A WHO grade I large cystic tumor that occurs almost exclusively in infants, with a prominent desmoplastic stroma having a neuroepithelial population consisting mainly of neoplastic astrocytes. It involves the superficial cerebral cortex and leptomeninges, and often attaches to the dura. Although clinically it presents as large tumor, it generally has a good prognosis following surgical resection. (Adapted from WHO) 